\title{
Perturbations of Gibbs Semigroups
}

\author{
V. A. Zagrebnov
}

Laboratory of Theoretical Physics, Joint Institute for Nuclear Research, SU-141980 Dubna, USSR

\begin{abstract}
We present the analytic perturbation theory for Gibbs semigroups in the case when perturbations of generators are relatively bounded. Analyticity with respect to perturbation and semigroup parameters in the Tr-norm topology is proved and the corresponding domains are described.
\end{abstract}

\section{Introduction}

Let $\mathfrak{C}_{p}(\mathfrak{H})$ be the Banach space of compact operators on a separable Hilbert space $\mathfrak{H}$ which have the finite $\|\cdot\|_{p}$-norm

$$
\|A\|_{p}=\left(\sum_{n=1}^{\infty} \lambda_{n}^{p}\right)^{1 / p}, \quad 1 \leqq p<\infty .
$$

Here $\lambda_{n}=\mu_{n}(|A|)$, where $\mu_{n}(|A|)$ is a $n^{\text {th }}$ (taking into account degeneracy) eigenvalue of the operator $|A|=\sqrt{A A^{*}}$. The Banach spaces $\left\{\mathfrak{C}_{p}(\mathfrak{G})\right\}_{p=1}^{\infty}$ are $*$-ideals in the space of all bounded operators $\mathscr{L}(\mathfrak{G})$ and $\mathfrak{C}_{1}(\mathfrak{G})$ (trace-class) $\subset \mathfrak{C}_{2}(\mathfrak{H})$ (HilbertSchmidt operators $) C \ldots \subset \mathfrak{C}_{\infty}(\mathfrak{H})$ (compact operators $) \subset \mathscr{L}(\mathfrak{H})$, see e.g. [1] or $[2$, VI.6].

In quantum statistical mechanics one faces strongly continuous oneparametric semigroups of self-adjoint operators $G: \mathbb{R}_{+}^{1} \cup\{0\} \rightarrow \mathscr{L}(\mathfrak{H})$ which have the property that $G: \mathbb{R}_{+}^{1} \rightarrow \mathfrak{C}_{1}(\mathfrak{H})$. They are naturally created by the density matrix $\exp (-\beta H)$ for a finite system with the Hamiltonian $H$ and temperature $\beta^{-1} \in \mathbb{R}_{+}^{1}$ and got the name of the Gibbs semigroups [3-5]. But if we want to make an analytic continuation in the "interaction constant" then the operator $H$ becomes non-selfadjoint. Its numerical range $\theta(H)=\{(H \psi, \psi): \psi \in D(H),\|\psi\|=1\}$ belongs to the sector $\mathscr{S}_{\gamma}(\Omega)=\{z \in \mathbb{C}:|\arg (z-\gamma)| \leqq \Omega<\pi / 2\}$ and $G(t)=\exp (-t H) \in \mathfrak{C}_{1}(\mathfrak{H})$ for $t \in \mathbb{R}_{+}^{1}$, see e.g. [6,7]. This was the reason for the following general definition.

Definition 1.1 [5]. A strongly continuous semigroup $G(t)$ in a separable Hilbert space $\mathfrak{S}$ is called a Gibbs semigroup if $G: \mathbb{R}_{+}^{1} \rightarrow \mathfrak{C}_{1}(\mathfrak{G})$. 
Remark 1.1. From the continuity of multiplication $\left(A_{n} B_{n} \stackrel{\|\cdot\|_{p}}{\longrightarrow} A B\right.$ if $A_{n} \stackrel{s}{\longrightarrow} A$ (strongly) and $B_{n} \stackrel{\|\cdot\|_{p}}{\longrightarrow} B$ for $\left.1 \leqq p<\infty[8,9]\right)$ and the fact that $\boldsymbol{C}_{1}(\mathfrak{H})$ is $*$-ideal in $\mathscr{L}(\mathfrak{S})$ one gets the $\|\cdot\|_{1}$-continuity of the Gibbs semigroup $G(t)$ on $\mathbb{R}_{+}^{1}$.

By the Hille-Yosida-Phillips theorem [9, X.8] each strongly continuous semigroup $V(t)$ is related to a closed operator $T$ (semigroup generator) with a dense domain $D(T)$, i.e., $V(t)=V_{T}(t)=\exp (-t T)$. The corresponding properties of the generator $T$ can be formulated using the resolvent $R_{\zeta}(T)=(\zeta-T)^{-1}$ and the resolvent set $P(T)=\left\{\zeta \in \mathbb{C}: R_{\zeta}(T) \in \mathscr{L}(\mathfrak{G})\right\}$. Therefore, the study of semigroups is mainly connected with properties of their generators, the central question being the problem of stability and perturbation theory.

Let $G_{T}(t)$ be a Gibbs semigroup with the generator $T$. The aim of the present paper is to develop a perturbation theory for the Gibbs semigroups. More precisely, we shall describe perturbations $U$ for which $G_{\lambda}(t)=G_{T+\lambda U}(t)$ is still the Gibbs semigroup $\|\cdot\|_{1}$-analytic with respect to the parameters $\{\lambda, t\}$.

In Sect. 2 we study $U$ from the Hille-Phillips perturbation class $\mathscr{P}_{0}$. This case has been earlier considered in refs. 3,4 . Here we simplify their proofs considerably and show that $\mathscr{P}_{0}$ consists of the operators $U$ which are $T$-bounded with the relative $T$-bound $b=0$. In this case the semigroup $G_{\lambda}(t)$ is $\|\cdot\|_{1}$-analytic on the parameter $\lambda$ in the whole complex plane $\mathbb{C}$.

In Sects. 3 and 4 we consider perturbations with $b>0$. The $\|\cdot\|_{1}$-analyticity in the parameter $\lambda$ requires that $b<1$ (class $\mathscr{P}_{1}$ ). Then the domain of $\|\cdot\|_{1}$-analyticity of the Gibbs semigroup $G_{\lambda}(z)$ has the form $C_{b} \times S_{\lambda, b}$. Here $C_{b}=\left\{z \in \mathbb{C}:|z|<(2 b)^{-1}\right\}$ and $S_{\lambda, b}$ is sector in the right half-plane $\mathbb{C}_{+}=\{z \in \mathbb{C}: \operatorname{Re} z>0\}$ :

$$
S_{\lambda, b}=\left\{z \in \mathbb{C}_{+}:|\arg z|<\omega=\operatorname{arctg} \frac{\sqrt{1-2|\lambda| b}}{|\lambda| b}\right\} .
$$

For perturbations from class $\mathscr{P}_{0}$ one correspondingly gets that a domain of the $\|\cdot\|_{1}$-analyticity of $G_{\lambda}(z)$ has the form $\mathbb{C} \times \mathbb{C}_{+}$.

Section 5 contains the concluding remarks and formulations of some other results of the Gibbs semigroup theory.

\section{Perturbations from Class $\mathscr{P}_{0}$}

The standard perturbation theory for strongly continuous semigroups has been developed by Hills and Phillips $[11, \mathrm{XIII}]$ for perturbations from class $\mathscr{P}_{0}$.

Definition 2.1. A closed operator $U$ belongs to the class $\mathscr{P}_{0}$ of perturbations for the generator $T$ of the semigroup $G_{T}(t)$ if the domain $D(U) \supseteq \bigcup_{t>0} G_{T}(t) \mathfrak{H}$ and

$$
\int_{0}^{1} d t\left\|U G_{T}(t)\right\|<\infty .
$$

We show that the Gibbs semigroups are stable with respect to perturbations $U \in \mathscr{P}_{0}$, the corresponding perturbation series converges in the $\|\cdot\|_{1}$-topology and defines a Gibbs semigroup. 
Theorem 2.1. Let $G_{T}(t)$ be a self-adjoint Gibbs semigroup with the generator $T \geqq-\alpha$. If the operator $U \in \mathscr{P}_{0}$, then the series

$$
\mathscr{F}_{\lambda}(t)=\sum_{n=0}^{\infty} \lambda^{n} S_{n}(t)
$$

with

$$
S_{n=0}(t)=G_{T}(t), \quad S_{n \geqq 1}(t)=-\int_{0}^{t} d \tau G_{T}(t-\tau) U S_{n-1}(\tau),
$$

(i) converges uniformly on $t$ from any compact $K \subset \mathbb{R}_{+}^{1}$ and on $\lambda$ from any disc $C \subset \mathbb{C}$ in the $\|\cdot\|_{1}$-topology;

(ii) defines the Gibbs semigroup $G_{\lambda}(t)$ with the generator $H_{\lambda}=T+\lambda U$ which is the $\|\cdot\|_{1}$-holomorphic function of the parameter $\lambda$ for any fixed $t>0$.

Proof. (i) The operator $U$ is $T$-bounded and $\mathfrak{C}_{1}(\mathfrak{H})$ is $*$-ideal in $\mathscr{L}(\mathfrak{H})$. Then, $U G_{T}(t)$ $=\left[U R_{\zeta}(T)\right]\left[(\zeta-T) G_{T}(t)\right] \in \mathfrak{C}_{1}(\mathfrak{H})$ for $t>0$ and $\zeta \in P(T)$. Together with condition (2.1) this implies that for $t>0$ the operator $S_{n}(t)$ can be represented as the $n$-fold $\|\cdot\|_{1}$-convergent Bochner integral:

$$
\begin{aligned}
S_{n}(t)= & \int_{0}^{t} d \tau_{1} \int_{0}^{t} d \tau_{2} \ldots \int_{0}^{t} d \tau_{n} \chi_{n}^{t}\left(\tau_{0}, \tau_{1}, \ldots, \tau_{n}\right) \\
& \times(-1)^{n} G_{T}\left(\tau_{0}\right) U G_{T}\left(\tau_{1}\right) \ldots U G_{T}\left(\tau_{n}\right) .
\end{aligned}
$$

Here $\chi_{n}^{t}\left(\tau_{0}, \tau_{1}, \ldots, \tau_{n}\right)$ is the characteristic function of the set $\left\{\tau_{i} \geqq 0 ; i=0,1, \ldots, n\right.$ : $\left.\sum_{i=0}^{n} \tau_{i}=t\right\}$. From the above representation one gets

$$
\begin{gathered}
\left\|S_{n}(t)\right\|_{1} \\
\leqq \int_{0}^{t} d \tau_{1} \ldots \int_{0}^{t} d \tau_{n} \chi_{n}^{t}\left(\tau_{0}, \tau_{1}, \ldots, \tau_{n}\right)\left\|G_{T}\left(\tau_{0}\right) U G_{T}\left(\tau_{1}\right) \ldots U G_{T}\left(\tau_{n}\right)\right\|_{1} .
\end{gathered}
$$

Now we can use the Ginibre-Gruber inequality [12]:

$$
\left\|\prod_{i=0}^{n} A_{i} V\left(x_{i}\right)\right\|_{1} \leqq\left(\prod_{i=0}^{n}\left\|A_{i}\right\|\right) \operatorname{Tr}\left[V\left(\sum_{i=0}^{n} x_{i}\right)\right]
$$

where $V(x)$ is an arbitrary Gibbs semigroup and $A_{i} \in \mathscr{L}(\mathfrak{H}), i=0,1, \ldots, n$. To this end we introduce $A_{0}=G_{T}\left(\tau_{0} / 2\right), A_{i}=U G_{T}\left(\tau_{i} / 2\right)$ for $i \geqq 1$ and the functions $q(t)$ $=\left\|G_{T}(t)\right\|=\exp \alpha t$ and $p(t)=\left\|U G_{T}(t)\right\|$. Then, using (2.4) and (2.5) one gets

$$
\left\|S_{n}(t)\right\|_{1} \leqq 2^{n}(q * \underbrace{p * \ldots * p}_{n})(t / 2) \operatorname{Tr} G_{T}(t / 2)
$$

where $*$ denotes convolution operation. From condition (2.1) it follows that for any $R>0$ there is $\varepsilon>0$ small enough, that $\int_{0}^{\varepsilon} d t\left\|U G_{T}(t)\right\|=\gamma_{\varepsilon}<(2 R)^{-1}$. Then, for $0<t \leqq 2 \varepsilon$ series (2.2) converges in the $\|\cdot\|_{1}$-topology uniformly on $\lambda$ from disc $C_{R}=\{z \in \mathbb{C}:|z|<R\}$ and on $t$ from any compact $K \subset(0,2 \varepsilon]$. In addition, from (2.2) 
one gets that

$$
\left\|\mathscr{F}_{\lambda}(t)\right\|_{1} \leqq \frac{(\exp \alpha t / 2-1)}{\alpha\left(1-2|\lambda| \gamma_{\varepsilon}\right)} \operatorname{Tr} G_{T}(t / 2)
$$

and that the operator-valued function $\mathscr{F}_{\lambda}(t)$ satisfies the equation (Duhamel formula $[10, \mathrm{X} .9])$ :

$$
\mathscr{F}_{\lambda}(t)=G_{T}(t)-\lambda \int_{0}^{t} d \tau G_{T}(t-\tau) U \mathscr{F}_{\lambda}(\tau)
$$

From Eq. (2.8) according to the standard arguments (see e.g. [13, XI]) it follows that for $0 \leqq t \leqq 2 \varepsilon$ the function $\mathscr{F}_{\lambda}(t)$ is a strongly continuous semigroup with the generator $H_{\lambda}=T+\lambda U$. Now we can extend the definition of $\mathscr{F}_{\lambda}(t)$ for $t \geqq 2 \varepsilon$ by putting

$$
\mathscr{F}_{\lambda}(t)=\left(\mathscr{F}_{\lambda}(\varepsilon)\right)^{n} \mathscr{F}_{\lambda}(t-n \varepsilon)
$$

if $n \varepsilon \leqq t \leqq(n+1) \varepsilon$. From the representation (2.9) and estimates (2.6), (2.7) one gets the uniform $\|\cdot\|_{1}$-convergence of series (2.2) for $t$ from any compact $K \subset \mathbb{R}_{+}^{1}$ and for $\lambda \in C_{R}$ for a disc of an arbitrary radius $R$.

(ii) The $\|\cdot\|_{1}$-convergence of (2.2) implies that the semigroup $\mathscr{F}_{\lambda}(t)$ for $t>0$ and $\lambda \in \mathbb{C}$ is a Gibbs semigroup, i.e. $\mathscr{F}_{\lambda}(t)=G_{\lambda}(t) \in \mathfrak{C}_{1}(\mathfrak{H})$, which is $\|\cdot\|_{1}$-continuous on $\mathbb{R}_{+}^{1}$ by Remark 1.1. Moreover, by construction of the series (2.2) the Gibbs semigroup $G_{\lambda}(t)$ is $\|\cdot\|_{1}$-holomorphic for $\lambda \in \mathbb{C}$ if $t>0$.

Corollary 2.1. Let the operator $U$ be symmetric. Then, the semigroup $G_{\lambda}(t)$ is the selfadjoint Gibbs semigroup for $t>0$ and $\lambda \in \mathbb{R}^{1}$. On the other hand, as it follows from the proof of Theorem 2.1 the statements (i) and (ii) hold if one substitutes the selfadjointness of the semigroup $G_{T}(t)$ with the generator $T \geqq-\alpha$ by a more general condition of its quasi-boundedness: $\left\|G_{T}(t)\right\| \leqq M \exp \alpha$, i.e. $G_{T}(t) \in \mathscr{B}(M, \alpha)$.

Corollary 2.2. Let $\mathscr{D} \subset \mathbb{C}$ and for any $\lambda \in \mathscr{D}$ the operator $U(\lambda)$ from the family $\{U(\lambda)\}_{\lambda \in \mathscr{D}}$ has the following properties:

(a) The operator $U(\lambda)$ is T-bounded: $\|U(\lambda) \psi\| \leqq a\|\psi\|+b\|T \psi\|, \psi \in D(T)$, and the function $\lambda \rightarrow U(\lambda) G_{T}(t)$ is $\|\cdot\|$-analytic for $t>0$;

(b) $\int_{0}^{1} d t \sup _{\lambda \in \mathscr{D}}\left\|U(\lambda) G_{T}(t)\right\|<\infty$.

Then, the operator-valued function $G_{T+U(\lambda)}(t)$ constructed by iterations of Eq. (2.8) is a Gibbs semigroup which is $\|\cdot\|_{1}$-analytic for $\lambda \in \mathscr{D}(t>0)$.

Note that conditions defining the perturbation class $\mathscr{P}_{0}$ are too severe. The next statement says that this class does not cover even perturbations arising in the quantum statistical mechanics $[14,15]$.

Theorem 2.2. Let $G_{T}(t)$ be a strongly continuous quasi-bounded semigroup with the generator $T$. If the operator $U \in \mathscr{P}_{0}$, then it is $T$-bounded with the relative bound $b=0$.

Proof. By the closedness of the operator $U$ and the property: $D(U) \supseteq \bigcup_{t>0} G_{T}(t) \mathfrak{H}$ we get that $U G_{T}(t) \in \mathscr{L}(\mathfrak{H})$ for $t>0$ and $\left\|U G_{T}(t)\right\| \leqq\left\|U G_{T}(t=1)\right\| M \exp [\alpha(t-1)]$ for 
$t>1$. Consequently, for an arbitrary small $\varepsilon>0$ one can find $\xi>\alpha$ large enough that

$$
\left\|\int_{0}^{\infty} d t \exp (-\xi t) U G_{T}(t) \psi\right\| \leqq \varepsilon\|\psi\|, \quad \psi \in \mathfrak{H} .
$$

Simultaneously for the semigroup $G_{T}(t)$ and for any $\zeta$ from the resolvent set $P(-T)$ one gets:

$$
\int_{0}^{\infty} d t \exp (-\zeta t) G_{T}(t) \psi=(\zeta+T)^{-1} \psi, \quad \psi \in \mathfrak{H} .
$$

This relation together with estimate (2.10) and the closedness of $U$ gives that $(\zeta+T)^{-1} \psi \in D(U)$ for $\psi \in \mathfrak{H}$ and $\|U \varphi\| \leqq \varepsilon \xi\|\varphi\|+\varepsilon\|T \varphi\|$ for $\varphi \in D(T)$, i.e., $b=0$.

The paper [7] is an attempt to develop a perturbation theory for the Gibbs semigroups when $b>0$. But it contains an error in the proof of the $\|\cdot\|_{1}$-analyticity of the semigroup $G_{\lambda}(t)$ on the parameter $\lambda$ and in the estimate of the domain of its $\|\cdot\|_{1}$-analyticity on the parameter $t$ in the continuation into the half-plane $\mathbb{C}_{+}$. Below, in Sects. 3 and 4, these errors will be corrected.

\section{Perturbations from Class $\mathscr{P}_{1}$}

As it follows from the above discussion a class of Gibbs semigroups which are stable with respect to perturbations from the class $\mathscr{P}_{0}$ contains all quasi-bounded Gibbs semigroups $\mathscr{B}(M, \alpha)$. We show that extension of the perturbations to the class $\mathscr{P}_{b}$ (operators which are relatively bounded by the generator with $b>0$ ) implies reduction of stable semigroups to the holomorphic Gibbs semigroups $\mathscr{H}(\omega, \alpha)$ with generators having the following property: $R_{\zeta}(T) \in \mathfrak{C}_{p}(\mathfrak{H})$ for $\zeta \in P(T)$ and some $p<\infty$. It is the class of semigroups that one encounters in quantum statistical mechanics [14-17].

Theorem 3.1. Let $G_{T}(t)$ be a self-adjoint Gibbs semigroup with the generator $T \geqq-\alpha$. Then, it admits an analytic continuation to the $\|\cdot\|_{1}$-holomorphic in the half-plane $\mathbb{C}_{+}$Gibbs semigroup $G_{T}(z) \in \mathscr{H}(\pi / 2, \alpha)$.

Proof. Using the spectral representation $G_{T}(t)=\int_{-\alpha}^{\infty} d E_{\xi}(T) \exp (-t \xi)$ one can check that $G_{T}(t>0) \mathfrak{G} \subseteq D(T)$. Therefore, the operator $T G_{T}(t) \in \mathscr{L}(\mathfrak{H})$ for $t>0$. Consequently, the semigroup $G_{T}(t>0)$ is strongly differentiable. By the Remark 1.1 this property can be lifted up to $\|\cdot\|_{1}$-differentiability

$$
\begin{aligned}
\|\cdot\|_{1}-\partial_{t} G_{T}(t) & =\|\cdot\|_{1}-\lim _{\Delta \rightarrow 0}\left\{\frac{1}{\Delta}\left[G_{T}(t / 2+\Delta)-G_{T}(t / 2)\right]\right\} G_{T}(t / 2) \\
& =(-T) G_{T}(t) \in \mathfrak{C}_{1}(\mathfrak{H}) .
\end{aligned}
$$

In the same way one gets for $t>0$ that

$$
\|\cdot\|_{1}-\partial_{t}^{n} G_{T}(t)=(-T)^{n} G_{T}(t) \in \mathfrak{C}_{1}(\mathfrak{H}), \quad n \geqq 1 .
$$


Then, due to the estimate $\left\|T G_{T}(t)\right\| \leqq \max \left\{t^{-1}, \alpha \exp \alpha t\right\}$, we obtain

$$
\left\|\partial_{t}^{n} G_{T}(t)\right\|_{1} \leqq\left\|T G_{T}(t / 2 n)\right\|^{n}\left\|G_{T}(t / 2)\right\|_{1} \leqq(2 n)^{n} t^{-n}\left\|G_{T}(t / 2)\right\|_{1} .
$$

Hence, the semigroup $G_{T}(t)$ can be continued from $\mathbb{R}_{+}^{1}$ to the disc $C_{t}=\left\{z \in \mathbb{C}_{+}:|z-t|<t / 2 e\right\}$ via the $\left\|_{1} \cdot\right\|_{1}$-convergent series

$$
G_{T}(z)=\sum_{n=0}^{\infty} \frac{(z-t)^{n}}{n !} \partial_{t}^{n} G_{T}(t)=\exp (-z T) .
$$

The union of these discs $\bigcup_{t>0} C_{t}$ is the sector

$$
\mathscr{S}_{0}(\Omega)=\left\{z \in \mathbb{C}_{+}:|\arg z|<\Omega=\arcsin (2 e)^{-1}\right\}
$$

in which one can continue the Gibbs semigroup $G_{T}(t)$ from $\mathbb{R}_{+}^{1}$ by the $\|\cdot\|_{1^{-}}$convergent power series. For a further $\|\cdot\|_{1}$-analytic continuation from the sector $\mathscr{S}_{0}(\Omega)$ to the half-plane $\mathbb{C}_{+}$one has to take into account that $G_{T}(z=t+i \tau)$ $=G_{T}(t) G_{T}(i \tau) \in \mathfrak{C}_{1}(\mathfrak{H})$ for $z \in \mathbb{C}$ and $\|\cdot\|_{1}-\partial_{z} G_{T}(z)=(-T) G_{T}(z) \in \mathfrak{C}_{1}(\mathfrak{H})$.

Definition 3.1. The closed operator $U$ belongs to the $\mathscr{P}_{1}$-class perturbations of the generator $T$ for a strongly continuous semigroup $G_{T}(t)$ if $D(U) \supset D(T)$ and $U$ is a $T$ bounded operator with a relative boundary $b<1$ :

$$
\|U \psi\| \leqq a\|\psi\|+b\|T \psi\|, \quad \psi \in D(T) .
$$

Remark 3.1. If, in addition, the operator $T$ is self-adjoint and semibounded from below and the operator $U$ is symmetric, then by the Kato-Rellich theorem $[10, \mathrm{X} .2],[13, \mathrm{~V}$, Sect. 4] the algebraic sum $H=T+U$ with $D(H)=D(T)$ is the selfadjoint operator and $H \geqq-\alpha^{\prime}=-\alpha-\max (a /(1-b) ; a+b|\alpha|)$.

Therefore, the operator $H$ is a generator of a strongly continuous (differentiable for $t>0)$ self-adjoint semigroup $V_{H}(t)$. From the same arguments as in Theorem 3.1 it can be extended to the $\|\cdot\|$-analytic in $\mathbb{C}_{+} \operatorname{semigroup~} V_{H}(z)$. If the operator $T$ is a generator of a Gibbs semigroup, then from Remark 3.1 and the Weyl min-max principle (see e.g. [18, XIII.1]) it follows that the spectrum of the operator $H$ is a pure point and $\mu_{n}(H) \geqq a+(1-b) \mu_{n}(T)$ for $n \geqq 1$ large enough. Hence $V_{I I}(t)$ is a Gibbs semigroup: $V_{H}(t)=G_{H}(t)$, and by Theorem 3.1 one gets that $G_{I I}(z) \in \mathscr{H}\left(\pi / 2, \alpha^{\prime}\right)$. Therefore, we have proved the following statement:

Theorem 3.2. Let $G_{T}(t)$ be a self-adjoint quasi-bounded Gibbs semigroup. Let perturbation $U \in \mathscr{P}_{1}$ and be a symmetric operator. Then the operator $H=T+U$ is a generator of the quasi-bounded self-adjoint Gibbs semigroup $G_{H}(t)$ which can be extended to the $\|\cdot\|_{1}$-holomorphic semigroup $G_{H}(z) \in \mathscr{H}\left(\pi / 2, \alpha^{\prime}\right)$.

Remark 3.2. Thus, self-adjoint Gibbs semigroups are stable with respect to perturbations from the class $\mathscr{P}_{1}$. Moreover, the conditions $D(U) \supset D(T)$ and $b<1$ can be relaxed. Indeed, one can require that the perturbation $U=U^{*}$ be semibounded from below and the algebraic sum $H=T+U$ be essentially self-adjoint on the domain $D(T) \cap D(U)$. These conditions allow us to use the Golden- 
Thompson inequality [18, XIII.17]

$$
\|\exp (-\tilde{H})\|_{1} \leqq\|\exp (-T / 2) \exp (-U) \exp (-T / 2)\|_{1},
$$

which says that the closure $(T+U)^{\sim}=\tilde{H}$ is a generator of the self-adjoint, semibounded Gibbs semigroup $G_{\tilde{H}}(t)$.

But as above (see Sect. 2), we would like in addition to stability of the selfadjoint Gibbs semigroups to study the analytic properties of the semigroup $G_{\lambda}(z)$ on the parameter $\lambda \in \mathbb{C}$ for perturbations $\lambda U \in \mathscr{P}_{1}$. Therefore, instead of the spectral representation for constructing the semigroup $G_{\lambda}(z)$ one has to exploit the Dunford-Taylor formula (see e.g. [2, VII] or [13, XI, Sect. 1.6]):

$$
G_{\lambda}(z)=\frac{1}{2 \pi i} \int_{\Gamma} d \zeta \exp (-\zeta z) R_{\zeta}\left(H_{\lambda}\right) .
$$

The right-hand side of (3.2) is the $\|\cdot\|$-convergent Bochner integral with a positively oriented contour $\Gamma \subset P\left(H_{\lambda}\right)$ with the spectrum of $H_{\lambda}$ contained within $\Gamma$. Formula (3.2) connects the properties of the semigroup $G_{T}(z)$ with that for a nonperturbed semigroup $G_{T}(z)$ by means of the resolvent $R_{\zeta}\left(H_{\lambda}\right)=\left(\zeta-H_{\lambda}\right)^{-1}$, $H_{\lambda}=T+\lambda U$.

Theorem 3.3. Let $T=T^{*} \geqq-\alpha$. Then for any $\lambda \in C_{b}=\left\{z \in \mathbb{C}:|z|<(2 b)^{-1}\right\}$ formula (3.2) defines the semigroup $G_{\lambda}(z)$ which is strongly analytic on the parameter $z$ in the sector $S_{\lambda, b}=\left\{z \in \mathbb{C}_{+}:|\arg z|<\omega=\operatorname{arctg}(\sqrt{1-2|\lambda| b} /|\lambda| b)\right\}$.

Proof. First of all we have to note that for $\lambda=0$ formula (3.2) gives the same result as Theorem 3.1. The line of reasoning is the following. For the operator $T$ the resolvent set is $P(T)=\{\mathbb{C} \backslash[-\alpha,+\infty)\}$. Therefore, for any $z \in \mathbb{C}_{+}$the contour $\Gamma=\Gamma_{0}$ can be chosen in such a way that $\operatorname{Re} z \operatorname{Re} \zeta>\operatorname{Im} z \operatorname{Im} \zeta$ for $\operatorname{Re} \zeta \rightarrow \infty$ (Fig. 1), i.e. the right-hand side of (3.2) is the $\|\cdot\|$-convergent Bochner integral. To verify that this integral defines a strongly holomorphic semigroup with the generator $T$

Fig. 1

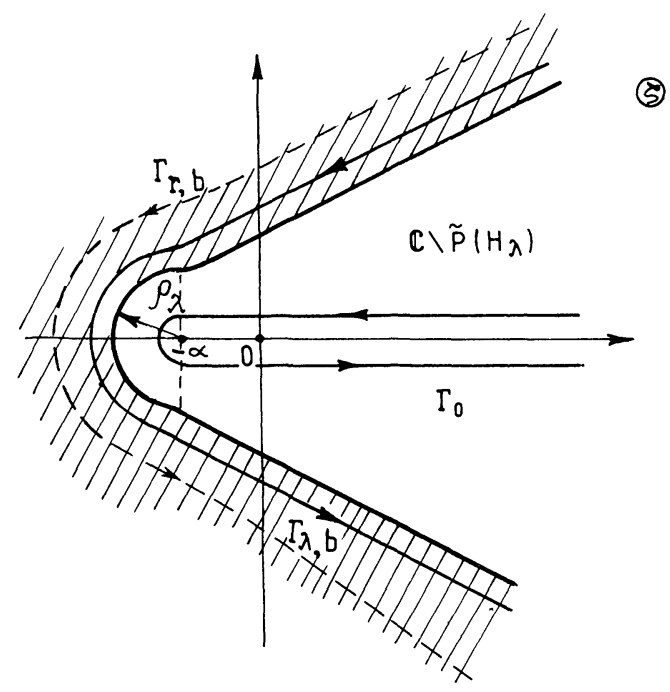


in the half-plane $\mathbb{C}_{+}$, one has to use the Cauchy theorem and differentiation under the integral (3.2). Now let perturbation $U \in \mathscr{P}_{1}$. Then, the operator $H_{\lambda}=T+\lambda U$ is well-defined as an algebraic sum and is closed on the domain $D\left(H_{\lambda}\right)=D(T)$ if $|\lambda| b<1$. The appropriate resolvent set $P\left(H_{\lambda}\right)$ is defined by the condition $|\lambda|\left\|U R_{\zeta}(T)\right\|<1$ corresponding to the convergence of the Neumann series for $R_{\zeta}\left(H_{\lambda}\right)$ :

$$
R_{\zeta}\left(H_{\lambda}\right)=R_{\zeta}(T) \sum_{n=0}^{\infty}\left(\lambda U R_{\zeta}(T)\right)^{n}
$$

Note that taking into account inequality (3.1) and the structure of the set $P(T)$ one gets

$$
\begin{array}{rlrl}
\left\|U R_{\zeta}(T)\right\| & \leqq a\left\|R_{\zeta}(T)\right\|+b\left\|T R_{\zeta}(T)\right\| & \\
& \leqq \begin{cases}\frac{a}{|\zeta+\alpha|}+b, & \operatorname{Re} \zeta<-\alpha \\
\frac{a}{\operatorname{Im}(\zeta+\alpha)}+b\left[1+\frac{|\zeta+\alpha|}{\operatorname{Im}(\zeta+\alpha)}\right], & \operatorname{Re} \zeta \geqq-\alpha .\end{cases}
\end{array}
$$

Then, from inequality (3.4) we obtain that the resolvent set $P\left(H_{\lambda}\right)$ contains as a subset

$$
\widetilde{P}\left(H_{\lambda}\right)= \begin{cases}|\zeta+\alpha|>\varrho_{\lambda}=\frac{a|\lambda|}{1-b|\lambda|}, & \operatorname{Re} \zeta<-\alpha \\ \operatorname{Im}(\zeta+\alpha)>\varrho_{\lambda}+\frac{|\lambda| b}{\sqrt{1-2|\lambda| b}} \operatorname{Re}(\zeta+\alpha), & \operatorname{Re} \zeta \geqq-\alpha .\end{cases}
$$

Therefore, the contour $\Gamma$ in the integral (3.2) can be chosen in $\widetilde{P}\left(H_{\lambda}\right)$, e.g. $\Gamma=\Gamma_{\lambda, b}$, see Fig. 1. Then, the integral convergence condition $(\operatorname{Re} z \operatorname{Re} \zeta>\operatorname{Im} z \operatorname{Im} \zeta$ for $\zeta \rightarrow \infty)$ defines a sector $S_{\lambda, b}$, where the semigroup $G_{\lambda}(z)$ is strongly analytic in $z$, see Fig. 2:

$$
S_{\lambda, b}=\left\{z \in \mathbb{C}_{+}:\left|\frac{\operatorname{Im} z}{\operatorname{Re} z}\right|<\frac{\sqrt{1-2|\lambda| b}}{|\lambda| b}\right\} .
$$

The semigroup and analytic properties of the function $z \rightarrow G_{\lambda}(z)$ can be verified similarly to the case $\lambda=0$. From (3.5) and (3.6) one gets that $G_{\lambda}(z) \in \mathscr{H}\left(\omega, \alpha^{\prime}(\lambda)\right)$,

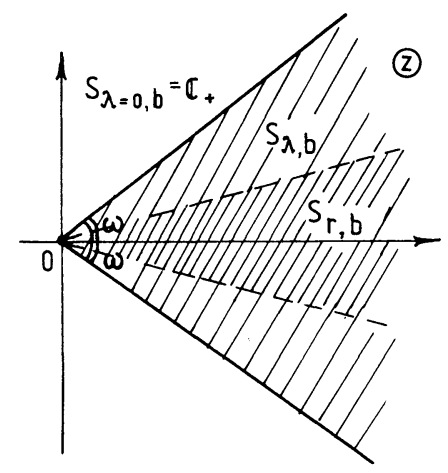


where $\alpha^{\prime}(\lambda) \geqq \alpha+a|\lambda| /(1-b|\lambda|)$ and $\omega=\operatorname{arctg}(\sqrt{1-2|\lambda| b} /|\lambda| b)$, see Fig. 2 .

Remark 3.3. If the operator $T$ is a generator of the Gibbs semigroup $G_{T}(t)$ and perturbation $U \in \mathscr{P}_{1}$ then the perturbed semigroup $G_{T}(t)$ for $\lambda \in C_{b}$ should not necessarily be Gibbsian. One needs supplementary conditions either on the operator $\lambda U$ (see Theorem 3.2 and Remark 3.2) or on the generator $T$. The latter will be considered in the next section.

\section{Gibbs Semigroups with $p$-Generators}

Let an unperturbed semigroup $G_{T}(t)$ be the Gibbs semigroup. For perturbations from the class $\mathscr{P}_{0}$ this guarantees that the semigroup $G_{\lambda}(t)$ for $\lambda \in \mathbb{C}$ will also be Gibbsian. Theorem 3.2 ensures the same for the semigroup $G_{T+U}(t)$ if the operator $U \in \mathscr{P}_{1}$ and is symmetric. Remark 3.2 makes clear the price one needs to pay for getting rid of the condition: $U \in \mathscr{P}_{1}$. But as before the symmetricity of the operator $U$ is essential. Since for perturbation $\lambda U$ with complex parameter $\lambda$ this property is violated, we start with conditions ensuring the stability of Gibbs semigroups with respect to these perturbations, see Remark 3.3.

Definition 4.1. We say that a self-adjoint Gibbs semigroup has p-generator $T$ if for some $\zeta_{0} \in P(T)$ the resolvent $R_{\zeta_{0}}(T) \in \mathfrak{C}_{p}(\mathfrak{H})$ for some finite $p \geqq 1$, see [7].

Remark 4.1. Let $T_{\sigma}\left(\Lambda^{N}\right)=\left[\sum_{1 \leqq i \leqq N}\left(-\Delta_{i} / 2 m\right)\right]_{\sigma}$ be the kinetic-energy operator of $N$ particles enclosed in a bounded vessel $\Lambda \subset \mathbb{R}^{d}$. Here $\sigma$ fixes a boundary condition on $\partial \Lambda$ corresponding to a self-adjoint extension of symmetric operator $T\left(\Lambda^{N}\right)$. Then one gets: $R_{\zeta_{0}}\left(T_{\sigma}\left(\Lambda^{N}\right)\right) \in \mathcal{C}_{p}(\mathfrak{H})$ for $p \geqq \frac{1}{2} \cdot N \cdot d$ and arbitrary $\zeta_{0} \in P\left(T_{\sigma}\left(\Lambda^{N}\right)\right)$.

Theorem 4.1. Let a self-adjoint operator $T \geqq-\alpha$ be a p-generator of the strongly continuous semigroup $G_{T}(t)$. Then, it is a $\|\cdot\|_{1}$-analytic in $\mathbb{C}_{+}$Gibbs semigroup. If the operator $U \in \mathscr{P}_{1}$, then a perturbed semigroup $G_{\lambda}(z)$ is also a Gibbs semigroup which is $\|\cdot\|_{1}$-analytic on $z$ in the sector $S_{\lambda, b}$ (3.6) for any $\lambda \in C_{b}=\{\zeta \in \mathbb{C}:|\zeta|$ $\left.<(2 b)^{-1}\right\}$.

Proof. For each $\zeta \in P(T)$ one gets: $R_{\zeta}(T)=R_{\zeta_{0}}(T)+\left(\zeta_{0}-\zeta\right) R_{\zeta}(T) R_{\zeta_{0}}(T)$. Thus, for an arbitrary $\zeta \in P(T)$ the resolvent $R_{\zeta}(T) \in \mathfrak{C}_{p}(\mathfrak{H})$ and the integral (3.2) along the path $\Gamma_{0}$ (Fig. 1) is the $\|\cdot\|_{p}$-convergent Bochner integral defining a semigroup $G_{T}(t) \in \mathfrak{C}_{p}(\mathfrak{H})$ for $t>0$. Then, the semigroup $G_{T}(t)=\left[G_{T}(t / p)\right]^{p} \in \mathfrak{C}_{1}(\mathfrak{H})$ and its $\|\cdot\|_{1^{-}}$ analyticity: $G_{T}(z) \in \mathscr{H}(\pi / 2, \alpha)$, can be verified in the same way as in Theorem 3.1. To estimate the $\|\cdot\|_{p}$-norm of the operator $G_{\lambda}(z)$ we use the expansion (3.3) and formula (3.2) for the case when the contour $\Gamma=\Gamma_{\lambda, b}$ (Fig. 1)

$$
\begin{aligned}
\left\|G_{\lambda}(z)\right\|_{p} \leqq & \frac{1}{2 \pi} \int_{\Gamma_{\lambda, b}}|d \zeta| \exp [-\operatorname{Re}(\zeta \cdot z)]\left\|R_{\zeta}(T)\right\|_{p}\left(1-|\lambda|\left\|U R_{\zeta}(T)\right\|\right)^{-1} \\
\leqq & \frac{1}{2 \pi} \int_{\Gamma_{\lambda, b}}|d \zeta| \exp [-(\operatorname{Re} \zeta \operatorname{Re} z-\operatorname{Im} \zeta \operatorname{Im} z)] \\
& \times\left\|R_{\zeta 0}(T)\right\| \frac{1+\left|\zeta_{0}-\zeta\right|\left\|R_{\zeta}(T)\right\|}{1-|\lambda|\left\|U R_{\zeta}(T)\right\|}
\end{aligned}
$$


Then, by Theorem 3.3 the right-hand side of (4.1) is bounded for $z \in S_{\lambda, b}$ and $|\lambda|<(2 b)^{-1}$, see (3.5) and (3.6). Consequently, the semigroup $G_{\lambda}(z)=\left[G_{\lambda}(z / p)\right]^{p} \in \mathfrak{C}_{1}(\mathfrak{H})$ and its $\|\cdot\|_{1}$-analyticity on $z$ in the sector $S_{\lambda, b}: G_{\lambda}(z) \in \mathscr{H}\left(\omega, \alpha^{\prime}(\lambda)\right)$ can be checked in the similar way as in Theorem 3.3.

Now we have to prove the $\|\cdot\|_{1}$-analyticity of the semigroup $G_{\lambda}(z)$ on the parameter $\lambda \in C_{b}=\left\{\zeta \in \mathbb{C}:|\zeta|<(2 b)^{-1}\right\}$ when $z \in S_{\lambda, b}$ (3.6). As a first step we prove the $\|\cdot\|_{p}$-analyticity.

Lemma 4.1. Let the operator $U \in \mathscr{P}_{1}$ and the generator of the semigroup $G_{T}(z)$ is a p-generator. Then, the Gibbs semigroup $G_{\lambda}(z)$ is $\|\cdot\|_{p}$-analytic on the parameter $\lambda$ in the disc $C_{b}$ (i.e. for an arbitrary closed domain $\mathscr{D} \subset C_{b}$ ) if $z \in \bigcap_{\lambda \in \mathscr{D}} S_{\lambda, b}$.

Proof. Let $r<(2 b)^{-1}$ be a fixed number and $z \in \bigcap_{|\lambda| \leqq r} S_{\lambda, b} \equiv S_{b}(r)$, see Fig. 2. Then, for all $\lambda$ such that $|\lambda| \leqq r$ one can pick out a common contour $\Gamma_{r, b} \subset \bigcap_{|\lambda| \leqq r} \widetilde{P}\left(H_{\lambda}\right)[\operatorname{see}(3.5)$ and Fig. 1] for the family of semigroups $\left\{G_{\lambda}(z)\right\}_{|\lambda| \leqq r}$ which ensures the $\|\cdot\|_{p^{-}}$ convergence of the integral (3.2) which can be estimated by (4.1). From the expansion (3.3) together with inequality (3.4) and estimate

$$
\begin{aligned}
& \left\|R_{\zeta}\left(H_{\lambda}\right)-R_{\zeta}(T) \sum_{n=0}^{N}\left(\lambda U R_{\zeta}(T)\right)^{n}\right\|_{p} \\
& \quad \leqq\left(1-|\lambda|\left\|U R_{\zeta}(T)\right\|\right)^{-1}\left\|R_{\zeta}(T)\right\|_{p}\left\|\lambda U R_{\zeta}(T)\right\|^{N+1},
\end{aligned}
$$

it follows that for $\zeta \in \Gamma_{r, b}$ the series (3.3) is uniformally convergent for $\lambda:|\lambda| \leqq r$, in the $\|\cdot\|_{p}$-norm. Hence, it defines a $\|\cdot\|_{p}$-analytic in this disc resolvent $R_{\zeta}\left(H_{\lambda}\right)$. Therefore, the series (3.3) can be term-by-term integrated by the contour $\Gamma_{r, b}$ for each $z \in \mathscr{D} \subset S_{b}(r)$ :

$$
G_{\lambda}(z)=\sum_{n=0}^{\infty} \lambda^{n} \frac{1}{2 \pi i} \int_{\Gamma_{r, b}} d \zeta \exp (-\zeta z) R_{\zeta}(T)\left(U R_{\zeta}(T)\right)^{n} .
$$

The series in the right-hand side of (4.2) converges uniformly in disc $C_{b}$ in $\|\cdot\|_{p^{-}}$ topology and defines a $\|\cdot\|_{p}$-analytic on the $\lambda \in C_{b}$ function which coincides with the Gibbs semigroup $G_{\lambda}(z)$.

Theorem 4.2. Let all conditions of Lemma 4.1 be satisfied. Then, the Gibbs semigroup $G_{\lambda}(z)$ is $\|\cdot\|_{1}$-analytic on $\lambda$ in the disc $C_{b}$ for each $z \in \bigcap_{\lambda \in \mathscr{D}} S_{\lambda, b}$.

Proof. By Lemma 4.1 the semigroup $G_{\lambda}(z)$ can be represented in the disc $C_{b}$ as the $\|\cdot\|_{p}$-convergent Taylor series

$$
G_{\lambda}(z)=\left.\sum_{n=0}^{\infty} \frac{\lambda^{n}}{n !} \partial_{\lambda}^{n} G_{\lambda}(z)\right|_{\lambda=0}, \quad z \in S_{\lambda, b} .
$$

Here, derivatives on $\lambda$ have the form

$$
\left.\partial_{\lambda}^{n} G_{\lambda}(z)\right|_{\lambda=0}=\frac{n !}{2 \pi i} \int_{\gamma} d \mu \mu^{-(n+1)} G_{\mu}(z)
$$


The contour $\gamma$ belongs to the disc $C_{b}$ and includes the point $\mu=0$, e.g. $\gamma=\gamma_{r}$, where $\gamma_{r}$ is a circle of the radius $r<(2 b)^{-1}$ centered at the origin. Then, by Theorem 4.1 one gets from (4.4) the following inequality:

$$
\left\|\left.\partial_{\lambda}^{n} G_{\lambda}(z)\right|_{\lambda=0}\right\|_{1} \leqq \frac{n !}{2 \pi} \int_{0}^{2 \pi} d \varphi r^{-n} \sup _{|\mu| \leqq r}\left\|G_{\mu}(z)\right\|_{1}=n ! r^{-n} M_{r}(z)
$$

and, as a consequence, the estimate:

$$
\left\|G_{\lambda}(z)-\left.\sum_{n=0}^{N} \frac{\lambda^{n}}{n !} \partial_{\lambda}^{n} G_{\lambda}(z)\right|_{\lambda=0}\right\|_{1} \leqq \sum_{n=N+1}^{\infty}|\lambda|^{n} r^{-n} M_{r}(z) .
$$

The estimate (4.5) implies for each $\lambda \in C_{b}$ and $z \in S_{\lambda, b}$ the existence of the contour $\gamma_{r}$ $C C_{b}$ such that the series (4.3) is $\|\cdot\|_{1}$-convergent uniformly in $\lambda:|\lambda| \leqq r$.

\section{Concluding Remarks}

Summarizing we can conclude that for perturbations of the Gibbs semigroups with the $p$-generators the mapping

$$
G_{\lambda}(z): C_{b} \times S_{\lambda, b} \rightarrow \mathfrak{C}_{1}(\mathfrak{H})
$$

is $\|\cdot\|_{1}$-analytic on both the parameters if only perturbations from the class $\mathscr{P}_{1}$ are involved, see Sects. 3 and 4.

If the relative bound $b \rightarrow 0$, then one gets from (5.1) the result of Sect. 2:

$$
G_{\lambda}(z): \mathbb{C} \times \mathbb{C}_{+} \rightarrow \mathbb{C}_{1}(\mathfrak{H}) .
$$

Although in this limit the generators of the unperturbed Gibbs semigroups need not be $p$-generators.

In quantum statistical mechanics the results (5.1) and (5.2) ensure the analyticity of the partition function

$$
Z_{\Lambda}(\lambda, \beta)=\operatorname{Tr} G_{\lambda}(\beta) ; \quad \lambda \in C_{b}, \quad \beta \in S_{\lambda, b}
$$

for a finite volume $\Lambda$. This property is a consequence of the $\|\cdot\|_{1}$-analyticity of the function $\operatorname{Tr}: \mathfrak{C}_{1}(\mathfrak{H}) \rightarrow \mathbb{C}$. It is often exploited in applications, e.g. for the proof of inequalities $[15,16]$ and of the theorems utilizing the convexity on $\lambda$ or $\beta[15-17]$.

The investigation of the Gibbs semigroups has been started in [4] where perturbation theory for the self-adjoint semigroups and bounded operators $U \in \mathscr{L}(\mathfrak{S})$ has been considered. An attempt to develop a consistent perturbation theory for unbounded operators $U$ is the intention of [6]. But it contains inaccuracies which are first of all connected with the incorrect use of the Duhamel formula for investigation of the $\|\cdot\|_{1}$-analytic properties of the semigroup $G_{\lambda}(t)$ on the parameter $\lambda$ see also $[17,2.4]$. In [3] the perturbation theory for the Gibbs semigroups has been developed for the operators $U \in \mathscr{P}_{0}$. In the present paper this class of perturbations is extended to $\mathscr{P}_{1}$ and the corresponding domains of the $\|\cdot\|_{1}$-analyticity, $C_{b}$ and $S_{\lambda, b}$, are analyzed in detail.

The $\|\cdot\|_{1}$-compactness of the families of Gibbs semigroups was considered in paper [5]. It is important for statistical mechanics of systems with singular potentials, see e.g. [14]. In the recent paper [19] the $\|\cdot\|_{1}$-convergent Trotter-Lie formula for Gibbs semigroup is discussed. 


\section{References}

1. Gohberg, I.C., Krein, M.G.: Introduction to the theory of linear nonself-adjoint operators. Transl. Math. Monographs, Vol. 18. Providence, RI: Am. Math. Soc. 1969

2. Reed, M., Simon, B.: Methods of modern mathematical physics, Vol. 1: Functional analysis. New York: Academic Press 1972

3. Angelescu, N., Nenciu, G., Bundaru, M.: On the perturbation of Gibbs semigroups. Commun. Math. Phys. 42, 29-30 (1975)

4. Uhlenbrock, D.: Perturbation of statistical semigroups in quantum statistical mechanics. J. Math. Phys. 12, 2503-2512 (1971)

5. Zagrebnov, V.A.: On the families of Gibbs semigroups. Commun. Math. Phys. 76, 269-276 (1980)

6. Zagrebnov, V.A., Brankov, J.G., Tonchev, N.S.: A rigorous statement about systems interacting with Boson field. Dokl. Acad. Nauk USSR 225, 71-73 (1975)

7. Maison, H.D.: Analyticity of the partition function for finite quantum systems. Commun. Math. Phys. 22, 166-172 (1971)

8. Grümm, H.R.: Two theorems about $\mathfrak{C}_{p}$. Rep. Math. Phys. 4, 211-215 (1973)

9. Simon, B.: Trace ideals and their applications. Lond. Math. Soc. Lecture Notes Ser., Vol. 35. Cambridge: Cambridge University Press 1979

10. Reed, M., Simon, B.: Methods of modern mathematical physics, Vol. II: Fourier analysis, Selfadjointness. New York: Academic Press 1975

11. Hille, E., Phillips, R.S.: Functional analysis and semigroups. Providence, R.I.: Am. Math. Soc. 1957

12. Ginibre, J., Gruber, C.: Green functions of the anisotropic Heisenberg model. Commun. Math. Phys. 11, 198-213 (1969)

13. Kato, T.: Perturbation theory for linear operators. Berlin, Heidelberg, New York: Springer 1976

14. Zagrebnov, V.A.: On singular potential interactions in quantum statistical mechanics. Trans. Moscow Math. Soc. 41, 101-120 (1980)

15. Ruelle, D.: Statistical mechanics. Rigorous results. New York: Benjamin 1969

16. Zagrebnov, V.A.: The approximating Hamiltonian method for an infinite-mode Dicke maser model with $A^{2}$-term. Z. Phys. B 55, 75-85 (1984)

17. Thirring, W.: A course in mathematical physics, Vol. 4: Quantum mechanics of large systems. New York, Wien: Springer 1980

18. Reed, M., Simon, B.: Methods of modern mathematical physics, Vol. IV: Analysis of operators. New York: Academic Press 1978

19. Zagrebnov, V.A.: The Trotter-Lie product formula for Gibbs semigroups. J. Math. Phys. 29, 888-891 (1988)

Communicated by Ya. G. Sinai

Received July 1, 1988 